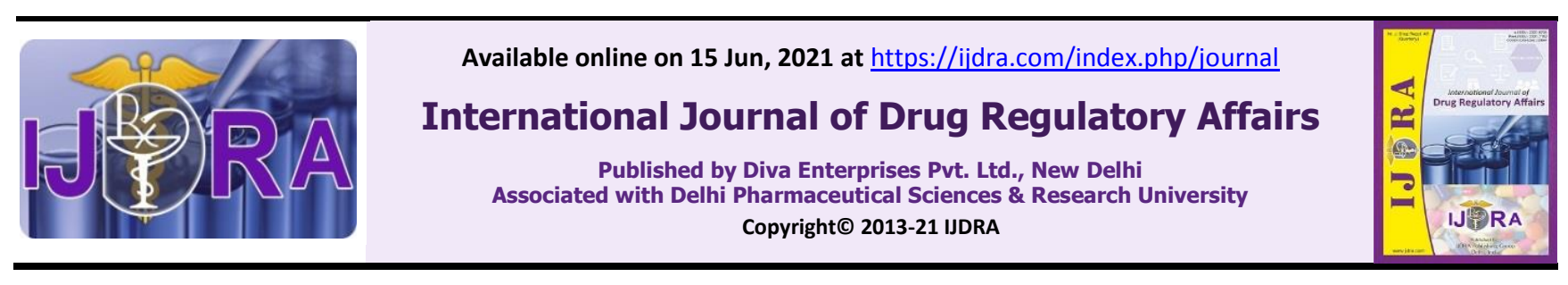

Review Article

OpenOAccess

\title{
Nitrosamine Impurities: Origin, Control and Regulatory Recommendations
}

\author{
Umesh Dobariya*, Narendra Chauhan, Himani Patel, Nidhi Pardeshi \\ Department of Regulatory Affairs, Isazi Pharma and Techno Consultancy Private Limited, Vadodara, Gujarat, India - 390007
}

\begin{abstract}
The unexpected finding of presence of nitrosamine impurities, by USFDA and EMA in year 2018, in drugs such as Angiotensin-II Receptor Blockers (ARBs), Ranitidine, Nizatidine and Metformin, has triggered the need for a risk assessment strategy for evaluation and control of these probable human carcinogen - nitrosamine in pharmaceutical product that are at risk. This finding leads to voluntarily recall of products worldwide. The finding of nitrosamines in some types of drug products led FDA and other international regulators to conduct a detailed risk assessment of these impurities in APIs and drug products. Although nitrosamine impurities have been found in only some drug products, regulatory agencies recommended to extend risk analysis in other chemically synthesized APIs and drug products also.
\end{abstract}

Keywords: Nitrosamine, Risk Assessment, USFDA, EMA, EDQM, Amines, Nitrous Acid, Azide

Article Info: Received 27 May. 2021; Review Completed 14 Jun. 2021; Accepted 15 Jun. 2021

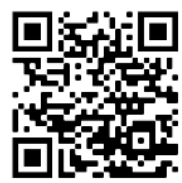

\section{Cite this article as:}

Dobariya U, Chauhan N, Patel H, Pardeshi N. Nitrosamine Impurities: Origin, Control and Regulatory Recommendations. Int J Drug Reg Affairs [Internet]. 2021 Jun 15 [cited 2021 Jun 15]; 9(2):77-80. Available from:

http://ijdra.com/index.php/journal/article/view/472

DOI: $10.22270 / i j d r a . v 9 i 2.472$

*Corresponding author

\section{Introduction}

In July 2018, health agencies were informed about presence of probable carcinogenic impurity identified as N-nitrosodimethylamine (NDMA) in Valsartan (Angiotensin-II Receptor Blockers (ARBs)). Since then, WHO, USFDA, EMA and other health agencies have been investigating the presence of nitrosamine impurities in certain drug products. Because the nitrosamine impurity issue is not limited to some region of world, USFDA and other regulatory authorities partnered to share investigation information, inspection coordination, communicate effective analytical methods to detect and identify various nitrosamines, and to develop rapid solutions to ensure the safety and quality of the drug supply. (1-4)

Regulators with which USFDA collaborated include the European Medicines Agency (EMA), European Directorate for the Quality of Medicines and Healthcare (EDQM), Health Canada (HC), Therapeutic Goods Administration (TGA, Australia), Ministry of Health, Labour and Welfare/ Pharmaceuticals and Medical Devices Agency (PMDA/MHLW, Japan), Health Sciences Authority, Singapore (HSA, Singapore), and Swissmedic (Switzerland). (1)

Nitrosamine are the molecule containing nitroso functional group and is of concern because these molecules are classified as probable carcinogens by
International Agency for Research on Cancer [IARC] based on animal studies. Nitrosamine are common in water and foods, cured and grilled meats, dairy products and vegetables. Importantly, formation of Nitrosamine in Medicinal products is process driven and not relate to any particular molecule. $(1,2)$

There are seven nitrosamine impurities (Figure 1) that could be present in drug products theoretically and five of them (NDMA, NDEA, NMBA, NIPEA, and NMPA) have actually been detected in drug substances or drug products. (1)

\section{Origin of Nitrosamine}

The most common pathway for the formation of Nitrosamine is Nitrosating reaction between amines (secondary, tertiary, or quaternary amines) and nitrous acid (nitrite salts under acidic conditions). Amines and nitrous acid can be present in the reaction process by various reasons.

Secondary, Tertiary and Quaternary Amines, present as a functional group in the API, intermediate and starting materials or added intentionally as a reagents or catalysts, can react with nitrous acid or other nitrosating agents to form nitrosamines.

Amide solvents, N-methylpyrrolidone, N,Ndimethylacetamide, and $\mathrm{N}, \mathrm{N}$-diethylacetamide are susceptible to degradation under certain reaction 
conditions to form secondary amines. Tertiary and quaternary amines used as reagents in the synthesis of APIs may contain other amine impurities. Secondary and tertiary amines may be present as impurities or degradants formed by dealkylation of quaternary amines.

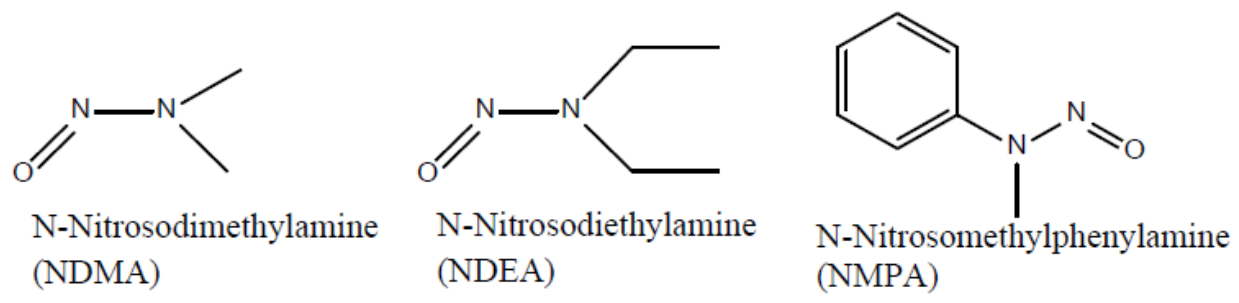

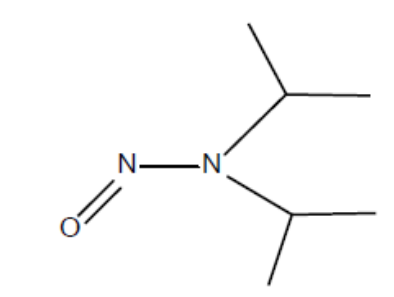

N-Nitrosodiisopropylamine (NDIPA)

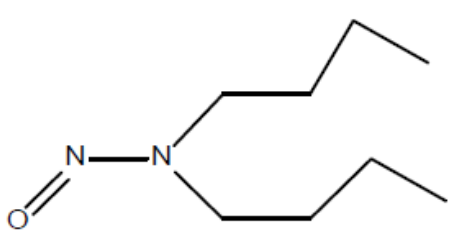

N-Nitrosodibutylamine (NDBA)

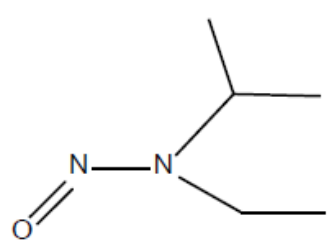

N-Nitrosoisopropylethylamine (NIPEA)<smiles>CN(CCCC(=O)O)N=O</smiles>

N-Nitroso-N-methyl-4-aminobutyric Acid (NMBA)

Figure 1. Structure of Different Nitrosamine impurities

NDMA can occur in drinking water as it is a by-product of several industrial processes and is a contaminant of certain pesticides. Nitrosamine impurities can be introduced when vendor-sourced materials, including starting materials and raw materials, are contaminated. The agency has observed incidents where starting materials and other raw materials are contaminated during shipment from vendors, nitrite impurities present in some starting materials and raw materials, secondary and tertiary amines present as impurities in some raw materials and fresh solvents, and cross-contamination of out-sourced starting materials and intermediates manufactured at sites where nitrosamine impurities are produced in other processes and equipment is not adequately cleaned.

Recovered materials such as solvents, reagents, and catalysts may pose a risk of nitrosamine impurities due to the presence of residual amines. These nitrosamines may be entrained if they have boiling points or solubility properties similar to the recovered materials, depending on how recovery and subsequent purification takes place.

Quenching process for decomposition of residual azide by addition of nitrous acid directly into reaction mixture, where nitrous acid, despite purification operations, can carry over into subsequent steps and comes into contact with residual amines in the raw materials and forms nitrosamine. Lack of optimization of the manufacturing process for APIs, where reaction conditions such as temperature, $\mathrm{pH}$, or the sequence of adding reagents, intermediates, or solvents are inappropriate or poorly controlled, is another potential source of formation of nitrosamine impurities.

Nitrites are common nitrosating impurities present in commonly used excipients which may lead to formation of nitrosamine impurities in drug product during manufacturing process as well as during storage. Processes that use nitrites in the presence of secondary, tertiary, or quaternary amines are at risk of generating nitrosamine impurities. Some drug products may undergo degradation pathways to form nitrosamine impurities during storage. $(1,2,4,7,8)$

\section{Controls Strategies and Regulatory Recommendations}

As per ICH M7(R1): Assessment and Control of DNA Reactive (Mutagenic) Impurities in Pharmaceuticals to Limit Potential Carcinogenic Risk (March 2018), control of any known mutagenic carcinogen, such as nitrosocompounds, at or below a level such that there would be a negligible human cancer risk associated with the exposure to potentially mutagenic impurities. (5) 
Regulatory agencies, including USFDA, EMA and EDQM, recommends that manufacturers consider the potential causes of nitrosamine formation and evaluate the risk for formation and contamination of nitrosamine impurities in their APIs and drug products based on factors such as maximum daily dose, duration of treatment, therapeutic indication, and number of patients treated. Manufacturers of APIs and drug products should take appropriate measures to prevent unacceptable levels of nitrosamine impurities in their products. (1-3)

Nitrosamines are not expected to be formed during the manufacture of the vast majority of APIs. However, it is now known that these impurities can form during production under certain conditions and when certain solvents, reagents and other raw materials are used. In addition, impurities can be carried over during the manufacturing process when using already-contaminated equipment or reagents. Furthermore, in cases where nitrosamines can form or are carried over during production, the impurities should normally be controlled and removed during the manufacturing process. Therefore, despite the low risk of nitrosamines being present, manufacturers were asked to take precautionary measures to mitigate the risk of nitrosamine formation or presence during the manufacture of all medicinal products containing chemically synthesised APIs. $(1,2)$

Active Pharmaceutical Ingredients Committee (APIC) recommended criteria of the risk assessment for presence of N-nitrosamines in APIs to prioritize the evaluations, as provided below. (6)

$>$ Higher daily dose taken

$>$ Long duration of treatment

$>$ Therapeutic indication

$>\quad$ Higher number of patients treated

$>$ Commercial APIs > APIs used for clinical trials

$>$ API manufactured in multipurpose equipment $>$ dedicated equipment

$>$ API manufactured in multipurpose equipment exposed to nitrosating agents

$>$ API > Intermediate > RSM (for companies manufacturing the three categories)

$>\quad$ APIs still manufactured $>$ APIs no longer manufactured but still on the market

$>$ APIs sold to markets where risk assessment has already been requested by authorities > APIs sold to other markets

$>\quad$ Knowledge of the likelihood of a risk based on the chemistry of the process (presence of amine, nitro functionalities, nitrosating agents)

USFDA recommends the acceptable intake (AI) limits for the nitrosamine impurities NDMA and NMBA as 96 ng/day and for NDEA, NMPA, NIPEA and NDIPA 26.5 ng/day. These limits are applicable only if a drug product contains a single nitrosamine. FDA suggested, if more than one nitrosamine impurities is detected and the total quantity of nitrosamine impurities exceeds 26.5 ng/day based on the maximum daily dose (MDD), the manufacturer should contact the Agency for evaluation. If nitrosamines without published acceptable intake limits are found in drug products, manufacturers should use the approach outlined in ICH M7(R1) to determine the risk associated with the nitrosamine and contact the Agency about the acceptability of any proposed limit. (1)

Sensitive methods with limits of quantitation (LOQ) in the parts-per-billion ( $\mathrm{ppb}$ ) range are needed to meet the low acceptable intake recommended for nitrosamines. Manufacturers should use methods with LOQs at or below $0.03 \mathrm{ppm}$. If more than one nitrosamine is detected, then the analytical method should be validated for LOQs below $0.03 \mathrm{ppm}$ to accurately quantify a total nitrosamine level of not more than $26.5 \mathrm{ng} /$ day. $(1,2)$

\section{Conclusion}

Manufacturer should identify potential sources for formation of Nitrosamine impurities such as raw material, reagent, catalyst, solvents and crosscontamination in chemically synthesized drug substance and drug products manufactured thereof. Regulatory agencies are publishing instruction notes and guidance periodically to guide manufacturers to identify and evaluate Nitrosamine in their product and control within its acceptance limit. Regulatory agencies are still collecting updated evaluation data from manufacturers to update recommendations provided in guidance as more information becomes available. All regulatory agencies and pharmaceutical manufacturers are working so closely with each other that elementary information about chemistry, origin, evaluation and control strategies are now well known for further assessment.

\section{Acknowledgements}

The authors are very much thankful to Isazi Pharma and Techno Consultancy Private Limited for their support and constant encouragement.

Financial Disclosure statement: The authors received no specific funding for this work.

\section{Conflict of Interest}

The authors declare that there is no conflict of interest regarding the publication of this article.

\section{References}

1. Control of Nitrosamine Impurities in Human Drugs, [Internet]. The United States Food and Drug Administration; 2021 Feb [cited 2021 May 01]. Available from:

https://www.fda.gov/media/141720/download

2. Nitrosamine impurities in human medicinal products [Internet]. European Medicines Agency; 2020 Jun [cited 2021 May 04]. Available from: https://www.ema.europa.eu/en/documents/referral/n itrosamines-emea-h-a53-1490-assessmentreport_en.pdf

3. The EDQM's Response to Nitrosamine Contamination [Internet]. The European Directorate for the Quality of Medicines \& HealthCare [cited 2021 May 01]. Available from:

https://www.edqm.eu/en/edqms-responsenitrosamine-contamination

4. WHO Information Note: Update on Nitrosamine impurities [Internet]. World Health Organization; 2020 Apr [cited 2021 May 04]. Available from: 
https://www.who.int/docs/default-

source/substandard-and-falsified/informationnotenitrosamine-impurities-april2020.pdf

5. ICH M7(R1) Guideline: Assessment and Control of DNA Reactive (Mutagenic) Impurities in Pharmaceuticals to Limit Potential Carcinogenic Risk [Internet]. The International Council for Harmonisation of Technical Requirements for Pharmaceuticals for Human Use; 2017 Mar [cited 2021 May 01]. Available from:

https://database.ich.org/sites/default/files/M7_R1_G uideline.pdf

6. Additional guidance on the assessment on the risk assessment for presence of $\mathrm{N}$-nitrosamines in APIs. [Internet]. Active Pharmaceutical Ingredients Committee (APIC) [cited 2021 May 01]. Available from:

https://www.apic.cefic.org/pub/APIC_Guidance_on _Nitrosamines_Risk_Assessment-final18Feb2020.pdf

7. Nitrosamine impurities in medications: Overview [Internet]. Health Canada; 2021 [cited 2021 May 05]. Available from:

https://www.canada.ca/en/healthcanada/services/drugs-health-products/complianceenforcement/information-healthproduct/drugs/nitrosamine-impurities.html

8. Evaluation of potential nitrosamines in connection with new authorisations [Internet], Swissmedic, 2020 [cited 2021 May 01]. Available from: https://www.swissmedic.ch/swissmedic/en/home/hu manarzneimittel/authorisations/information/bewertu ng_potentieller_nitrosamine_nas.html 